PROCEEDINGS OF THE

AMERICAN MATHEMATICAL SOCIETY

Volume 133, Number 2, Pages 415-423

S 0002-9939(04)07578-1

Article electronically published on August 4, 2004

\title{
HYPERBOLIC UNIT GROUPS
}

\author{
S. O. JURIAANS, I. B. S. PASSI, AND DIPENDRA PRASAD
}

(Communicated by Jonathan I. Hall)

\begin{abstract}
In this paper we study the groups $\mathcal{G}$ whose integral group rings have hyperbolic unit groups $\mathcal{U}(\mathbb{Z} \mathcal{G})$. We classify completely the torsion subgroups of $\mathcal{U}(\mathbb{Z G})$ and the polycyclic-by-finite subgroups of the group $\mathcal{G}$. Finally, we classify the groups for which the boundary of $\mathcal{U}(\mathbb{Z} \mathcal{G})$ has dimension zero.
\end{abstract}

\section{INTRODUCTION}

The study of hyperbolic groups has been an active topic of research in recent years. It started with the work of M. Gromov [4] and has developed very rapidly since then.

Let $\mathcal{G}$ be a group and $\Gamma:=\mathcal{U}_{1}(\mathbb{Z} \mathcal{G})$ the group of normalized units of the integral group ring $\mathbb{Z} \mathcal{G}$. It is well known that if $\mathcal{G}$ is finite, then $\Gamma$ is finitely presented (see, for instance, [8]). Since almost every finitely presented group is hyperbolic [10, it is natural to investigate when $\Gamma$ is hyperbolic. Motivated by these considerations, we are led to pose the following:

Problem 1. Classify the groups $\mathcal{G}$ for which $\Gamma$ is hyperbolic.

This paper is a contribution to the above problem. After giving in section 2 the basic facts about hyperbolic groups needed in this work, we characterize, in section 3, the torsion subgroups of $\Gamma$ and the polycyclic-by-finite subgroups of $\mathcal{G}$, thus answering the problem, in particular, for torsion groups. Contrary to the theorem that almost all finitely presented groups are hyperbolic, we find that the unit group of a finite group is hyperbolic only in a very small number of cases, which we enumerate explicitly. It turns out that if $\mathcal{G}$ is finite and $\Gamma$ is hyperbolic, then $\mathcal{G}$ has a normal free complement (i.e., there exists a normal free subgroup $F$ in $\Gamma$ such that $\Gamma=F \mathcal{G}, F \cap \mathcal{G}=1$ ), and furthermore every torsion-free complement of $\mathcal{G}$ is free.

Finally, we completely characterize the groups $\mathcal{G}$ such that $\Gamma$ is hyperbolic with its hyperbolic boundary having dimension zero, or equivalently that $\Gamma$ is virtually free.

Jespers ([6], [7]) has classified those finite groups $\mathcal{G}$ that have a normal free complement in $\Gamma$. This property implies that $\Gamma$ is quasi-isometric to a free group of finite rank and hence is hyperbolic. In view of this work, our Theorems 2 and

Received by the editors March 20, 2003 and, in revised form, October 18, 2003.

2000 Mathematics Subject Classification. Primary 20C07, 16S34, 20F67.

Key words and phrases. Hyperbolic group, group ring, unit group, Wedderburn decomposition.

This research was partially supported by CNPq-Brazil, FAPESP-Brazil. 
3 can be considered to be basically due to Jespers; however, our proofs are quite different.

\section{Preliminaries}

Let $(X, d)$ be a metric space. For $x, y, z \in X$, the Gromov product of $y, z$ with respect to $x$ is defined to be

$$
(y . z)_{x}=\frac{1}{2}\{d(y, x)+d(z, x)-d(y, z)\} .
$$

The metric space is said to be $\delta$-hyperbolic $(\delta \geq 0)$ if

$$
(x . y)_{w} \geq \min \left\{(x . z)_{w},(y . z)_{w}\right\}-\delta
$$

for all $w, x, y, z \in X$. Let $G$ be a finitely generated group and $S$ a finite set of generators for $G$. The Cayley graph $\mathcal{G}(G, S)$ of $G$ with respect to $S$ is the metric graph whose vertices are in one-to-one correspondence with the elements of $G$ and which has an edge (labeled $s$ ) of length 1 joining $g$ to $g s$ for each $g \in G$ and $s \in S$. The group $G$ is said to be hyperbolic (in the sense of Gromov) if its Cayley graph $\mathcal{G}(G, S)$ is a $\delta$-hyperbolic metric space for some $\delta \geq 0$. This definition does not depend on the choice of the generating set $S$.

For the reader's convenience, we collect some of the facts about hyperbolic groups that we need. Let $\mathbb{Z}^{2}$ denote the free Abelian group of rank two.

Theorem 1. Let $\Gamma$ be a hyperbolic group. Then

(a) $\mathbb{Z}^{2}$ does not embed as a subgroup of $\Gamma$.

(b) If $g \in \Gamma$ has infinite order, then $\left[C_{\Gamma}(g):\langle g\rangle\right]$ is finite, where $C_{\Gamma}(g)$ is the centralizer of $g$ in $\Gamma$.

(c) Torsion subgroups of $\Gamma$ are finite of bounded order.

(d) $\Gamma$ is virtually free if and only if its boundary has dimension zero.

(e) If $\Gamma$ is quasi-isometric to a free group, then $\Gamma$ is virtually free. If, moreover, $\Gamma$ is torsion-free, then it is free.

For the theory of hyperbolic groups, the reader may refer to [1, 3] or [4] and, for standard results and notation in group rings, to [11, 12, 13, 14.

\section{Groups with $\mathcal{U}_{1}(\mathbb{Z G})$ Hyperbolic}

Let $\mathcal{G}$ be an arbitrary group, and let $G \subseteq \Gamma:=\mathcal{U}_{1}(\mathbb{Z G})$ be a subgroup of normalized units. In this section, unless otherwise stated, we shall always assume that $\Gamma$ is a hyperbolic group.

We first make some easy observations:

$\mathbf{1}^{0}$. In case $G$ is finite, then it constitutes a $\mathbb{Z}$-linearly independent subset of $\mathbb{Z G}$ and consequently $\mathbb{Z}[G]$, the subring of $\mathbb{Z} \mathcal{G}$ generated by $G$, is isomorphic to the group ring $\mathbb{Z} G$. This fact shall be used freely. It is a simple consequence of a well-known theorem of Berman: If $u$ is an element of finite order in the unit group $\mathcal{U}_{1}(\mathbb{Z} \mathcal{G})$, then the coefficient in $u$ of the identity element of $\mathcal{G}$ is zero unless $u$ equals 1 .

$\mathbf{2}^{0}$. Let $g \in \Gamma$ be a torsion element and $n=o(g)$ its order. Since the torsionfree rank of $\mathcal{U}_{1}(\mathbb{Z}\langle g\rangle)$ is $\sum_{d \mid n, d>2}\left(\frac{\phi(d)}{2}-1\right.$ ) (see [13], Theorem 3.1, p. 54) where $\phi$ is the Euler phi-function, it follows, from Theorem $1(\mathrm{a})$, that $n$ divides 5,8 , or 12 . 
$3^{0}$. Let $G$ be a finite subgroup of $\Gamma$. Since $\mathbb{Z}^{2}$ does not embed in $\Gamma, \mathbb{Q} G$ has at most one Wedderburn component that is not a division ring, and that component must be $M_{2}(\mathbb{Q})$. (This follows by looking at the upper triangular matrices in a Wedderburn component $M_{n}(D)$, which gives rise to units of the form $G L_{n}(\mathcal{O})$ for an order $\mathcal{O}$ in $D$.) Hence if $G$ is non-Abelian and none of its non-Abelian quotient embeds into a division ring, then $\Delta\left(G, G^{\prime}\right)$, the kernel of the natural projection $\mathbb{Q} G \longrightarrow \mathbb{Q}\left(G / G^{\prime}\right)$, where $G^{\prime}$ is the derived group of $G$, is isomorphic to $M_{2}(\mathbb{Q})$, and so its dimension over $\mathbb{Q}$ is 4 .

$4^{0}$. In view of $\mathbf{2}^{0}$ and $\mathbf{3}^{0}$, it is easy to see that the groups $C_{5} \times C_{3}, C_{5} \times C_{4}, C_{5} \times C_{5}$, $D_{5}, C_{5} \rtimes C_{4}, C_{5} \rtimes C_{8}, C_{8} \times C_{2}, K_{8} \times C_{3},\left(C_{2} \times C_{2}\right) \rtimes C_{4}$ and $Q_{16}$ cannot appear as subgroups of $\Gamma$, where $Q_{16}$ is the generalized quaternion group of order $16, K_{8}$ denotes the quaternion group of order $8, D_{n}$ the dihedral group of order $2 n$ and $C_{n}$ the cyclic group of order $n$.

$5^{0}$. We will have occasion to use the general result that if $\Gamma:=\mathcal{U}_{1}(\mathbb{Z G})$ is finitely generated, so is $\mathcal{G}$; in particular if $\Gamma$ is hyperbolic, $\mathcal{G}$ is finitely generated. We supply a proof of this assertion. Suppose that $\Gamma$ is generated by a finite set, say $u_{1}, \cdots, u_{n}$. Let $G_{0}$ be the subgroup of $\mathcal{G}$ generated by the $\operatorname{supp}\left(u_{i}\right), \operatorname{supp}\left(u_{i}^{-1}\right), i=1, \cdots, n$. This is a finitely generated group. All the $u_{i}$ 's belong to the unit group of $\mathbb{Z} G_{0}$, and hence $\Gamma$ is contained in the unit group of $\mathbb{Z} G_{0}$. But $\mathcal{G}$ is a subset of $\Gamma$, and so $\mathcal{G}$ is contained in $\mathbb{Z} G_{0}$. This implies that $\mathcal{G}=G_{0}$, and hence $\mathcal{G}$ is finitely generated, as $G_{0}$ is.

Recall that a non-Abelian group $G$ is called Hamiltonian if all its subgroups are normal. We will abuse terminology to denote a non-Abelian, non-Hamiltonian group simply as a non-Hamiltonian group; thus in our usage, a non-Hamiltonian group is always non-Abelian. If $G$ is a finite Hamiltonian group, then $G \simeq K_{8} \times$ $A \times E$, where $K_{8}$ is the quaternion group of order $8, A$ an Abelian group of odd order and $E$ an elementary Abelian 2-group (see [5], Theorem 7.12, p. 308). As in [6], we begin with the following result.

Lemma 1. Let $H \subseteq G \subseteq \Gamma$ be groups with $G$ finite and non-Abelian. Then one of the following holds:

(a) $H$ is Abelian.

(b) $H$ is a (non-Abelian) Hamiltonian 2-group.

(c) $\mathbb{Q} H$ contains a unique matrix Wedderburn component that is isomorphic to $M_{2}(\mathbb{Q})$ and $H=G$.

Proof. Note first that if $M_{n}(D)$ is a Wedderburn component of $\mathbb{Q} H$, then we must have $n \leq 2$.

Suppose $H$ is non-Abelian. In case $\mathbb{Q} H$ is a direct sum of division rings, then $H \simeq K_{8} \times E \times A$, with $E$ an elementary Abelian 2-group and $A$ an Abelian group of odd order (13], Theorem 1.17, p. 172). In case $A \neq 1$, then, because of the restriction on the orders of the elements of finite order, $A$ is an elementary Abelian 3 -group. Hence $H_{0} \simeq K_{8} \times C_{3}$ is a subgroup of $G$, which is not possible by $4^{0}$, and so $H$ is a Hamiltonian 2-group.

Next suppose that $\mathbb{Q} H$ is not a direct sum of division rings. Then, by $3^{0}, \mathbb{Q} H$ has a unique matrix Wedderburn component $\mathcal{A}$ and $\mathcal{A} \simeq M_{2}(\mathbb{Q})$. Let $e \in \mathbb{Q} H$ be the primitive central idempotent such that $\mathbb{Q} H e=\mathcal{A}$. Let $\left\{f_{i} \mid 1 \leq 1 \leq n\right\}$ be the 
set of primitive central idempotents of $\mathbb{Q} G$, and so $e=\sum_{e f_{i} \neq 0} e f_{i}$. Since $\mathcal{A}$ is simple, $e f_{i} \neq 0$ implies that $\mathbb{Q} G f_{i}$ contains a copy of $\mathcal{A}$ and hence, since the only matrix Wedderburn component in $\mathbb{Q} G$ is $M_{2}(\mathbb{Q}), \mathbb{Q} G f_{i} \simeq M_{2}(\mathbb{Q})$. Also, there is exactly one index $i$, such that $e f_{i}$ is non-zero; for, otherwise, there will be more than one Wedderburn component in $\mathbb{Q} G$ which is $M_{2}(\mathbb{Q})$, which is not allowed by $\mathbf{3}^{0}$. So $e=e f_{i}$ and $\mathbb{Q} H e=\mathbb{Q} G e$ for a central primitive idempotent $e$ in $H$. We conclude from this that $H=G$. For this, let $g \in G$; thus $g e \in \mathbb{Q} H e$. Since the trace of a primitive central idempotent in a group ring, i.e., the coefficient of 1 , is non-zero, $g$ belongs to the support of $g e$, which is contained in $H$. Thus $G$ is contained in $H$, and hence $G=H$.

Corollary 1. Let $G$ be a finite non-Hamiltonian subgroup of $\Gamma$. Then every Wedderburn component of $\mathbb{Q} G$ is either $\mathbb{Q}$, or an imaginary quadratic extension of $\mathbb{Q}$, or $M_{2}(\mathbb{Q})$ or a totally definite quaternion algebra over $\mathbb{Q}$. Furthermore, $G$ has a subgroup of index 2 .

Proof. By the previous lemma there exists a unique Wedderburn component of the form $M_{2}(\mathbb{Q})$. Let $\mathcal{A}$ be any other Wedderburn component. Then $\mathcal{A}$ is a division ring. Let $K$ be a maximal subfield of $\mathcal{A}$. Then the unit group of the ring of integers of $K$ must be finite, and hence $K$ is at most a quadratic extension of $\mathbb{Q}$. So if $\mathcal{A}$ is non-commutative, then its centre must equal $\mathbb{Q}$, and hence $\operatorname{dim}_{\mathbb{Q}}(\mathcal{A})=4$, and thus $\mathcal{A}$ is a totally definite quaternion algebra over $\mathbb{Q}$.

Considering the complex group algebra $\mathbb{C} G$ and using what we proved above about the Wedderburn components of $\mathbb{Q} G$, we see that $\mathbb{C} G$ is the direct sums of copies of $\mathbb{C}$ and two-by-two matrices over $\mathbb{C}$. Hence, by Corollary 12.9 of [2], $G$ contains a subgroup of index 2 .

We note that if $G$ is a finite non-Hamiltonian subgroup of $\Gamma$ (and therefore has a Wedderburn component that is not a division algebra), then $\mathbb{Z} G$ does not contain a central unit of infinite order; for, if $\alpha$ is such an element, then for a $\theta$ in $\mathbb{Q} G$, a non-zero nilpotent element with $\theta^{2}=0,\langle\alpha, 1+\theta\rangle \cong \mathbb{Z}^{2}$, a contradiction to Theorem 1(a). Hence central units of $\mathbb{Z} G$ are trivial. The following result extends this observation to arbitrary subgroups of $\Gamma$.

Lemma 2. Let $G$ be any subgroup of $\Gamma$ such that $\mathcal{U}_{1}(\mathbb{Z}[G])$ contains a central unit of infinite order. If $G$ is finite, then it is Abelian; if $G$ is infinite and contained in $\mathcal{G}$, then $G=\mathcal{U}_{1}(\mathbb{Z} G)$ and $G$ is centre-by-finite.

Proof. If $G$ is finite, then by Lemma 1 and the above observation $G$ must be Abelian.

Suppose that $G$ is an infinite subgroup of $\mathcal{G}$ and $\alpha$ is a central unit in $\mathbb{Z} G$ of infinite order. Then, since $\Gamma$ is hyperbolic, by Theorem $1(b)$ we have that $\left[\mathcal{U}_{1}(\mathbb{Z} G):\langle\alpha\rangle\right]<$ $\infty$. It follows that $G$ is an elementary hyperbolic group with a central element $g_{o}$, say, of infinite order. If $0 \neq \theta$ is a nilpotent element in $\mathbb{Z} G$, then $\left\langle 1+\theta, g_{0}\right\rangle \simeq \mathbb{Z}^{2}$, since the Kaplansky trace of a nilpotent element is zero. Hence $\mathbb{Z} G$ has no non-zero nilpotent elements. For an element $x$ of $G$ of order $d$, and an arbitrary element $g$ of $G$, the element $(1-x) g\left(1+x+\cdots+x^{d-1}\right)$ is nilpotent, hence is zero. From this we see that the element $g$ normalizes the cyclic subgroup generated by $x$ for every torsion element $x$ in $G$. Thus the torsion elements $T(G)$ of $G$ form a subgroup of $G$, which is finite by Theorem $1(c)$. Furthermore, since all elements of $\mathcal{U}_{1}(\mathbb{Z} T(G)$ ) will have to be of finite order, $\mathcal{U}_{1}(\mathbb{Z} T(G))=T(G)$. Since $G / T(G)$ is ordered it follows from $\left([14]\right.$, Proposition 45.5, p. 277) that $\mathcal{U}_{1}(\mathbb{Z} G)=\left(\mathcal{U}_{1}(\mathbb{Z} T(G))\right) G=G$. 
Lemma 3. Let $G$ be any group, and let $x, y \in G$ be such that $\langle x\rangle \cap\langle y\rangle=1$, $o(x)<\infty, o(y) \geq 5$ and $x^{y} \notin\langle x\rangle$. Then $\mathbb{Z}^{2}$ embeds into $\mathcal{U}_{1}(\mathbb{Z} G)$.

Proof. Define $\hat{x}:=1+x+\cdots+x^{n-1}, n=o(x)$, and $\theta_{k}=(1-x) y^{k} \hat{x}, 1 \leq k \leq o(y)$. Now observe that, under the given hypothesis, it is possible to choose $k$ such that $\left\langle 1+\theta_{1}, 1+\theta_{k}\right\rangle$ is a subgroup of $\mathcal{U}_{1}(\mathbb{Z} G)$ and isomorphic to $\mathbb{Z}^{2}$.

Lemma 4. If $\Gamma$ has an element of order 5 , then $\mathcal{G} \simeq C_{5}$.

Proof. By general properties of elements of prime order in a group ring (see [14, Theorem 45.11, p. 278), if $\Gamma$ has an element of order 5 , so does $\mathcal{G}$. Let $x \in \mathcal{G}$ be such an element.

Suppose first that $\mathcal{G}$ is non-Abelian. If $x$ were central, then $\Gamma$ would have a central element $\alpha$ of infinite order, with support in $\langle x\rangle$, and hence, by Lemma 2 $\mathcal{G}$ would have an element $g_{0}$ of infinite order. Since $\left\langle\alpha, g_{o}\right\rangle \simeq \mathbb{Z}^{2}$, we have a contradiction. On the other hand, if $\langle x\rangle$ were normal but not central, then there would exist an element $g_{0} \in \mathcal{G}$ with $o\left(g_{0}\right) \in\{2,4,8, \infty\}$ such that $\langle x\rangle \rtimes\left\langle g_{o}\right\rangle$ is a subgroup of $G$. In view of $\mathbf{4}^{0}, o\left(g_{0}\right)=\infty$ and with $\alpha$ as above, we have $\left\langle\alpha, g_{0}^{4}\right\rangle \simeq \mathbb{Z}^{2}$, again a contradiction. Hence there must exist another element $y \in \mathcal{G}$ of order 5 that does not commute with $x$; but then $x$ and $y$ satisfy the conditions of Lemma 3 which is a contradiction.

Next suppose that $\mathcal{G}$ is Abelian. Then, as seen above, $\mathcal{G}$ must be torsion and hence finite. From rank considerations of $\Gamma$, it is clear that $\mathcal{G} \simeq C_{5}$.

Lemma 5. If $\mathcal{G}$ is a non-torsion group, then $T(\mathcal{G})$ is a finite Hamiltonian group and $\mathcal{U}_{1}(\mathbb{Z} T(\mathcal{G}))=T(\mathcal{G})$. Moreover, the primitive central idempotents of $\mathbb{Q} T(\mathcal{G})$ are central in $\mathbb{Q} \mathcal{G}$.

Proof. Let $x, y \in \mathcal{G}$, with $o(x)<\infty=o(y)$. Then, by Lemma 3 we must have that $x^{y} \in\langle x\rangle$. Since the orders of the torsion elements of $\mathcal{G}$ divide 8 or 12 , it follows that $y^{4}$ must centralize $T(\mathcal{G})$, the set of torsion elements of $\mathcal{G}$. Let $y_{0}=y^{4}$, and let $z \in \mathcal{G}$ be any other torsion element. If $z$ does not normalize $\langle x\rangle$, then $(1-x) z \hat{x}$ and $y_{0}(1-x) z \hat{x}$ are $\mathbb{Q}$-linearly independent commuting nilpotent elements, and so $\mathbb{Z}^{2}$ embeds into $\Gamma$, a contradiction. It follows that $T(\mathcal{G})$ is a subgroup, is locally finite, and hence, since $\Gamma$ is hyperbolic, it is finite. If $\mathcal{U}_{1}(\mathbb{Z} T(\mathcal{G}))$ is not trivial, then, since $y_{0} \in \mathcal{G}$ has infinite order, we can embed $\mathbb{Z}^{2}$ into $\Gamma$. Finally, let $e \in \mathbb{Q} T(\mathcal{G})$ be a central primitive idempotent that is not fixed by $g \in \mathcal{G}$. Then $o(g)=\infty$ and so $\left\langle 1+e g, 1+y_{0} e g\right\rangle \simeq \mathbb{Z}^{2}$, a contradiction

Lemma 6. Let $G$ be a finite non-Abelian subgroup of $\Gamma$. Then $\exp (G)$ divides 12 and $G=\langle H, x\rangle$, where $H$ is a subgroup of index 2 and $x$ is a 2-element. Furthermore, (i) if 3 divides $|G|$, then $G$ is isomorphic either to $S_{3}$ or to $Q_{12}$; (ii) if $G$ is a 2-group having a non-central element of order 2 , then $G \simeq D_{4}$.

Proof. In view of Corollary 1, observe that $G$ has a subgroup $H$ of index 2 that is either Abelian or a Hamiltonian 2-group and its order is not divisible by 5 . So we may choose a 2-element $x \in G$ such that $x^{2} \in H$ and $G=\langle H, x\rangle$.

Suppose that 3 divides $|G|$; then $H$ must be Abelian. If $S y l_{3}(G)$, the Sylow 3 -subgroup of $G$, were central, then, by Lemma $1, G=\operatorname{Syl}_{3}(G) \times \operatorname{Syl}_{2}(G)$ with $\operatorname{Syl}_{2}(G)$ a Hamiltonian 2-group, and so $G \simeq C_{3} \times K_{8}$, a contradiction to $4^{0}$. Hence there exists $a \in H$ of order 3 such that $a^{x} \neq a$. Then, since clearly $\left(a^{-1} a^{x}\right)^{x}=$ $\left(a^{-1} a^{x}\right)^{-1}$, we have that $G \simeq C_{3} \rtimes\langle x\rangle$. We only need to rule out the case when 
$o(x)=8$. In this case $\mathbb{Q}\left(G / G^{\prime}\right) \simeq \mathbb{Q} C_{8}$, and so $\mathcal{U}_{1}(\mathbb{Z} G)$ has a central element of infinite order. Therefore, by Lemma 2$] G$ cannot be a subgroup of $\Gamma$.

Suppose next that $G$ is a 2-group and $y \in G$ is a non-central element of order 2. Suppose first that $y \in H$. Then, by Lemma 1, $G=\langle x, y\rangle$. Since $x^{2} \in H$, it follows that $\left[y, x^{2}\right]=1$ and so $G^{\prime}=\left\langle y y^{x}\right\rangle$. Since such a group does not possess a non-Abelian quotient that embeds in a non-commutative division ring, it follows by $3^{0}$ that $\operatorname{dim}_{\mathbb{Q}}\left(\Delta\left(G, G^{\prime}\right)\right)=4$ and so $|G|=8$; it thus follows that $G \simeq D_{4}$. Suppose that $y \notin H$. Then $G=\langle H, y\rangle$. Choose $a \in H$ such that $[a, y] \neq 1$. Then $G=\langle a, y\rangle$. Note that $z:=a^{-1} a^{y}$ is inverted by $y$. If it is not fixed, then $G=\langle z, y\rangle=\langle z\rangle \rtimes\langle y\rangle$. On the other hand, if $z$ is fixed, then $G^{\prime}=\langle z\rangle$ and the same argument as given above shows that $|G|=8$. Thus in any case it follows, by $3^{0}$, that $G \simeq D_{4}$.

It remains to show that $G$ has no element of order 8. Suppose $y \in G$ is an element of order 8. Then, in view of the previous paragraph, elements of order two are central. Let $z$ be such an element. If $z \notin\langle y\rangle$, then $C_{8} \times C_{2}$ would embed in $G$, which is not the case. Hence $z \in\langle y\rangle$, and thus $z=y^{4}$. Hence $G$ has a unique element of order 2. Since $G$ is not Abelian, it follows that $G$ is isomorphic to $Q_{16}$, which is ruled out by $\mathbf{4}^{0}$.

We are now ready to present our main results.

Theorem 2. Let $G$ be a finite non-Hamiltonian group. Then the following are equivalent:

(1) Exactly one Wedderburn component of $\mathbb{Q} G$ is $M_{2}(\mathbb{Q})$, and any other component is either $\mathbb{Q}$, or an imaginary quadratic extension of $\mathbb{Q}$ or a totally definite quaternion algebra over $\mathbb{Q}$.

(2) $G$ has a normal free complement in $\mathcal{U}_{1}(\mathbb{Z} G)$.

(3) $\mathcal{U}_{1}(\mathbb{Z} G)$ is virtually free.

(4) $\mathcal{U}_{1}(\mathbb{Z} G)$ is hyperbolic.

Moreover, if one of the above conditions holds, then every finitely generated torsion-free subgroup of $\mathcal{U}_{1}(\mathbb{Z} G)$ is free. In particular, any normal torsion-free complement of $G$ in $\mathcal{U}_{1}(\mathbb{Z} G)$ is free.

Proof. $(1) \Rightarrow(2)$ : From (1) it easily follows that c.d.(G), the set of complex character degrees of $G$, is $\{1,2\}$; hence, by Corollary 12.9 of $[2], G$ is metabelian. Furthermore, (1) also implies that $\mathbb{Q}\left(G / G^{\prime}\right)$ is a direct sum of copies of $\mathbb{Q}$ and imaginary quadratic fields. Hence the exponent of $G / G^{\prime}$ divides 4 or 6 , and so $\mathcal{U}_{1}\left(\mathbb{Z}\left(G / G^{\prime}\right)\right)$ is trivial. Therefore $F:=\mathcal{U}_{1}(\mathbb{Z} G) \cap\left(1+\Delta(G) \Delta\left(G^{\prime}\right)\right)$, which is known to be torsion-free (see [14; for a more general result see [9]), is a complement of $G$ in $\mathcal{U}_{1}(\mathbb{Z} G)$. Since $S L(2, \mathbb{Z})$ contains a free group of rank 2 as a subgroup of finite index, it easily follows that $F$ is quasi-isometric to a free group and so, by Theorem $1(\mathrm{e}), F$ is a free group.

$(2) \Rightarrow(3)$ : This implication is trivial, since $G$ is finite.

$(3) \Rightarrow(4)$ : This is a consequence of the fact that a free group is hyperbolic and hyperbolicity is stable under quasi-isometry.

$(4) \Rightarrow(1)$ : This follows from Corollary 1.

Finally, if $H$ is a finitely generated torsion-free subgroup of $\mathcal{U}_{1}(\mathbb{Z} G)$ and $F$ is a free subgroup of finite index in $\mathcal{U}_{1}(\mathbb{Z} G)$, then $H \cap F$ is a finitely generated free subgroup of finite index in $H$. Hence, once again by Theorem $\amalg(e)$, we have that $H$ is free. 
The following result characterizes the torsion groups that can occur as subgroups of hyperbolic unit groups.

Theorem 3. If a torsion group $G$ embeds into a hyperbolic unit group, then $G$ must be finite and isomorphic to one of the following groups:

(1) $C_{5}, C_{8}, C_{12}$, an Abelian group of exponent dividing 4 or 6 ;

(2) a Hamiltonian 2-group;

(3) $S_{3}, D_{4}, Q_{12}, C_{4} \rtimes C_{4}$.

Conversely, all of the groups listed above have hyperbolic unit groups.

Proof. Since a torsion subgroup of a hyperbolic group is finite, $G$ must be so.

Suppose first that $G$ is Abelian. Write

$$
\mathbb{Q} G=\bigoplus a_{d} \mathbb{Q}\left(\xi_{d}\right)
$$

where $a_{d}=\frac{n_{d}}{\phi(d)}, n_{d}=$ the number of elements of order $d$, and $\phi$ is the Euler phifunction. Then $\mathcal{U}_{1}(\mathbb{Z} G)$ is hyperbolic if and only if its torsion-free rank is at most one, i.e.,

$$
\sum_{d} a_{d}\left(\frac{\phi(d)}{2}-1\right) \leq 1
$$

Hence either $\mathcal{U}_{1}(\mathbb{Z} G)$ is finite, and so $G$ has exponent dividing 4 or 6 , or there exists a unique integer $d$ such that $\phi(d)=4$ and $a_{d}=1$. It then follows that $G \in\left\{C_{5}, C_{8}, C_{12}\right\}$.

Clearly, if $G$ is one of these groups, then the unit group of $\mathcal{U}_{1}(\mathbb{Z} G)$ is either trivial or has torsion-free rank equal to one and so is hyperbolic.

Suppose $G$ is non-Hamiltonian. In view of Lemma 6, we can suppose that $G$ is a 2-group of order at least 16 in which all elements of order 2 are central.

Since $G$ is non-Hamiltonian, we may choose $a, x \in G$ such that $a^{x} \notin\langle a\rangle$ and so, by Lemma 1, $G=\langle a, x\rangle$ and $[a, x] \neq a^{2}$. If $x^{a} \in\langle x\rangle$, then $G \simeq C_{4} \rtimes C_{4}$. So we also suppose that $x^{a} \notin\langle x\rangle$. Let $\bar{G}=G /\left\langle a^{2}\right\rangle$; then the Wedderburn components of $\mathbb{Q} \bar{G}$ are among those of $\mathbb{Q} G$. Hence, by Theorem $2, \bar{G}$ embeds into a hyperbolic unit group. Since the image of $a$ in $\bar{G}$ is not central, it follows, by Lemma 6 , that $\bar{G} \simeq D_{4}$. So $G$ has order 16 and we may choose a non-central element $y \in G$ whose image has order 4 in $\bar{G}$. We still have that $G=\langle y, x\rangle$ and either $[y, x]=y^{2}$ or $[y, x]=y^{2} x^{2}$. In particular, $[y, x]=[x, y]$. If $[y, x]=y^{2}$, then $G \simeq C_{4} \rtimes C_{4}$. On the other hand, if $[y, x]=y^{2} x^{2}$, then $x y$ would be a non-central element of order 2 and so, by Lemma 6. $G$ would have order 8, which is not the case.

If $G$ is Hamiltonian, then, by Lemma 6 , $G$ is a 2-group.

For the converse, since the unit group of a Hamiltonian 2-group is trivial, it only remains to show that the groups in (3) have hyperbolic unit groups. To do so, we prove that for all these groups Theorem 2(1) is satisfied. Indeed, for all of them we have that $G / G^{\prime}$ has exponent dividing 4 , and so $\mathbb{Q}\left(G / G^{\prime}\right)$ is a direct sum of copies of $\mathbb{Q}$ and $\mathbb{Q}(\sqrt{-1})$. Also, for all of them, $\operatorname{dim}_{\mathbb{Q}}\left(\Delta\left(G, G^{\prime}\right) \leq 8\right.$. Each of the groups $Q_{12}$ and $C_{4} \rtimes C_{4}$ embeds into a division ring (see [15], Theorem 2.1.5, p. 47). Because of the limitation on the dimension, these division rings are four dimensional over $\mathbb{Q}$ and hence are totally definite quaternion algebras, and the proof is complete by Theorem 2 .

We next characterize the infinite polycyclic-by-finite groups $G$ that embed in a group $\mathcal{G}$ whose unit group is hyperbolic. 
Theorem 4. An infinite polycyclic-by-finite group $G$ embeds into a group $\mathcal{G}$ whose unit group $\mathcal{U}_{1}(\mathbb{Z G})$ is hyperbolic if and only if

(1) $T(G)$, the set of elements of finite order in $G$, is a subgroup of $G$;

(2) $G \simeq T(G) \rtimes \mathbb{Z}$;

(3) $\mathcal{U}_{1}(\mathbb{Z} T(G))=T(G)$.

Proof. Let $G$ be infinite and polycyclic-by-finite; then $G$ has an element of infinite order and so, by Lemma $5, T(G)$ is a finite subgroup and $\mathcal{U}_{1}(\mathbb{Z}(T(G)))=T(G)$. Since $G$ is polycyclic-by-finite, it contains a normal free Abelian subgroup $A$, say. Since $\mathcal{U}_{1}(\mathbb{Z} \mathcal{G})$ is hyperbolic, it follows that $A=\langle x\rangle$ is cyclic. Applying Theorem 1(b), it is easy to see that $[G:\langle x\rangle]<\infty$ and so $G$ has Hirsch length one and thus, $G \simeq T(G) \rtimes \mathbb{Z}$. Since the hypothesis of ([14, Proposition 45.5, p. 277) is satisfied, we conclude that $\mathcal{U}_{1}(\mathbb{Z} G)=\left(\mathcal{U}_{1}(\mathbb{Z}(T(G))) G=(T(G)) G=G\right.$. The converse being trivial, the proof is complete.

Finally, we give a complete characterization of the groups $\mathcal{G}$ whose unit group $\Gamma$ is finitely generated virtually free, or equivalently the boundary $\partial(\Gamma)$ has dimension zero 4. For convenience we adopt the following:

Definition 1. A group $\mathcal{G}$ is called a $*$-group if one of the following conditions holds.

(1) $\mathcal{G}$ is a finite Abelian group of exponent dividing 4 or 6 .

(2) $\mathcal{G}$ is a finite Hamiltonian 2-group.

(3) $\mathcal{G} \in\left\{C_{5}, C_{8}, C_{12}, S_{3}, D_{4}, Q_{12}, C_{4} \rtimes C_{4}\right\}$.

(4) $\mathcal{G}=H \rtimes F$, where $H$ is of type (1) or (2) above and $F$ is a finitely generated free group.

Theorem 5. The unit group $\Gamma=\mathcal{U}_{1}(\mathbb{Z} G)$ of a group $G$ is finitely generated virtually free if and only if $G$ is a *-group. Furthermore, in case $G$ is infinite, $\Gamma=G$.

Proof. Suppose that $\Gamma$ is finitely generated virtually free, i.e., there exist a finitely generated free group $F$ contained in $\Gamma$ of finite index. Then $F$ is finitely generated and $\Gamma$ is hyperbolic. Hence if $G$ is a finite group, then by Theorem 3 is of type (1), (2) or (3). So suppose that $G$ is infinite. By $\mathbf{5}^{0}$, it follows that $G \cap F$ is a finitely generated free subgroup of $G$ of finite index in $G$, and thus $G$ is virtually free and so is hyperbolic. Since $G$ must necessarily be non-torsion, therefore, by Lemma 5 we have that $T(G)$ is finite and $\mathcal{U}_{1}(\mathbb{Z} T(G))=T(G)$. The quotient group $H:=G /(T(G))$ is quasi-isometric to the free group $G \cap F$, both being quasi-isometric to $G$; therefore, by Theorem 1(e), $H$ is virtually free. Since $H$ is torsion-free, it follows that $H$ is free. Hence $G \simeq T(G) \rtimes H$. Since $H$ is ordered and $\mathcal{U}_{1}(\mathbb{Z} T(G))=T(G)$, it follows from Proposition 45.5 of 14 that $\Gamma=G$. So in any case $G$ is a $*$-group.

The converse follows by the previous results and ([14], Prop. 45.5).

\section{ACKNOWLEDGMENTS}

The first author would like to thank the Harish-Chandra Research Institute, Allahabad (India) for its warm hospitality during his visit in February, 2003. The second author would like to thank the Instituto de Matemática e Estatística, São Paulo (Brazil) for its warm hospitality during his visit in September-October, 2002. 


\section{REFERENCES}

[1] Coornaert, M., Delzant, T., Papadopoulos, A. : Géométrie et théorie des groupes, LNM 1441, Springer-Verlag, 1990. MR 92f:57003

[2] Isaac, I.M. : Character Theory of Finite Groups, Academic Press, New York, 1976. MR $57: 417$

[3] Ghys, E. and Harpe, P. de la (Editors) : Sur les Groupes Hyperboliques d'après Mikhael Gromov, Progr. Math. Vol. 83, Birkhäuser, 1990. MR 92f:53050

[4] Gromov, M. : Hyperbolic groups, In: Essays in group theory (S. M. Gersten, Ed.), Springer Verlag, MSRI Publ. 8, 1997, 75-263. MR 89e:20070

[5] Huppert, B. : Endliche Gruppen I, Springer-Verlag, 1967. MR 37:302

[6] Jespers, E. : Free normal complements and the unit group of integral group rings, Proc. AMS vol. 122 (1994), 59-66. MR 94k:16058

[7] Jespers, E. : Units in integral group rings: a survey, Methods in Ring Theory, Lecture Notes in Pure and Applied Mathematics, 198, Dekker, New York, (1998), 141-169. MR 2001d:16048

[8] Kleinert, E. : Units of classical orders: a survey, L'Enseignement Math. 40 (1994), 205-248. MR 95k:11151

[9] Marciniak, Z., and Sehgal S. K. : The unit group of $1+\Delta(G) \Delta(A)$ is torsion free, J. Group Theory 6 (2003), 223-228. MR 2004a:16043

[10] Ol'Shanski1̌, A.Yu. : Almost every group is hyperbolic, Internat. J. Algebra Comput. 2 (1992), 1-17. MR 93j:20068

[11] Passi, I.B.S. : Group Rings and Their Augmentation Ideals, Lecture Notes in Mathematics 715, Springer, New York, 1979. MR 80k:20009

[12] Passman, D.S. : Algebraic Structure of Group Rings, Interscience, New York, 1977. MR 81d:16001

[13] Sehgal, S.K. : Topics in Group Rings, Marcel Dekker, New York and Basel, 1978. MR 80j:16001

[14] Sehgal, S.K. : Units in Integral Group Rings, Longman, Essex, 1993. MR 94m:16039

[15] Shirvani, M., Wehrfritz, B.A.F. : Skew Linear Groups, London Math. Soc. Lecture Notes Series 118, Cambridge, 1986. MR 89h:20001

Instituto de Matemática e Estatística, CP. 666.281, CEP.05315-970, SÃo Paulo, Brazil E-mail address: ostanley@ime.usp.br

Harish-Chandra Research Institute, Chhatnag Road, Jhunsi, Allahabad 211019, India

E-mail address: passi@mri.ernet.in

School of Mathematics, Tata Institute of Fundamental Research, Homi Bhabha Road, Colaba, Mumbai 400005, India

E-mail address: dprasad@math.tifr.res.in 